\title{
Resection versus Radiofrequency Thermal Ablation of Colorectal Liver Metastasis: A Retrospective Comparative Study
}

\author{
Mohamed Said Ghali, MD, MRCS; Wael A Jumah, MD; Ahmed Gamal El-Din Osman, MD, \\ MRCS \\ Department of General Surgery, Ain Shams University, Cairo, Egypt
}

Hepatic resection (HR) has been reported as the standard treatment for colorectal liver metastasis. Nevertheless, HR might not be suitable for some situations where those patients might be helped by chemoembolization or radiofrequency ablation (RFA) to treat their disease. RFA was first introduced in the late 1990s, however, it is questionable that RFA has similar survival rates as HR for patients with resectable liver metastasis. The aim of the present study was to compare the survival rates in patients with colorectal liver metastasis who were offered RFA versus HR.

Patients and methods: This was a retrospective study conducted on 40 patients, 16 underwent RFA and 24 underwent HR for colorectal liver metastasis. Kaplan-Meier curves were used to determine survival and the log-rank test was used for comparison between groups.

Results: The 3-year overall survival rates for patients with HR and RFA were 51.5 and $44.1 \%$ respectively $(p=0.285)$.

Conclusion: The 3-year overall and disease-free survival with RFA were similar to those treated with HR.

Key words: Colorectal cancer, liver metastasis, radiofrequency ablation, hepatic resection.

\section{Introduction}

Hepatic resection (HR) had been reported as the standard treatment for colorectal liver metastasis. ${ }^{1,2}$ Nevertheless, HR might be difficult with unfavorable tumor location, insufficient hepatic reserve, or having co-morbidities. Those patients could be candidates for chemoembolization or radiofrequency ablation (RFA).3,4 RFA was first introduced in the late 1990s and it was described as a minimally invasive procedure having low morbidity and short hospital stay for unresectable colorectal liver metastase ${ }^{5-7}$ and could be used repeatedly for recurrence. ${ }^{8,9}$

The aim of this study was to compare the survival rates of patients having liver metastasis who were offered RFA versus HR.

\section{Patients and methods}

This was a retrospective study conducted on 40 patients with colorectal liver metastasis who were admitted at Ain Shams University hospitals, Cairo, Egypt in the period between January 2009 and December 2015. The study was approved by the ethical and scientific committee of the General Surgery Department, Ain-Shams University. Those patients were diagnosed to have maximum 3 lesions of colorectal liver metastasis with a size of less than or equal to $3 \mathrm{~cm}$. The presence of liver metastasis was confirmed by preoperative abdominal computed tomography (CT), intraoperative diagnosis, or postoperative follow- up CT. Exclusion criteria were unresected primary tumors, distant metastasis to other organ, multiple metastases, and incomplete follow-up. The medical records of the 40 patients were analyzed retrospectively. Percutaneous RFA was performed in cases having co-morbidities, patients refusing surgery, or in cases of inadequate liver reserve. Patients were divided into two groups. One group was offered HR ( $n=24)$ with treatment assignment (5 right hepatectomies (Figure 1), 9 left hepatectomies (Figure 2), 4 segmentectomies, and 6 non-anatomical resections), whereas the other group was offered RFA $(n=16)$, RFA was performed percutaneously under local anesthesia using ultrasonographic guidance to ensure that at least a $1-\mathrm{cm}$ ablation margin was achieved around the tumor.

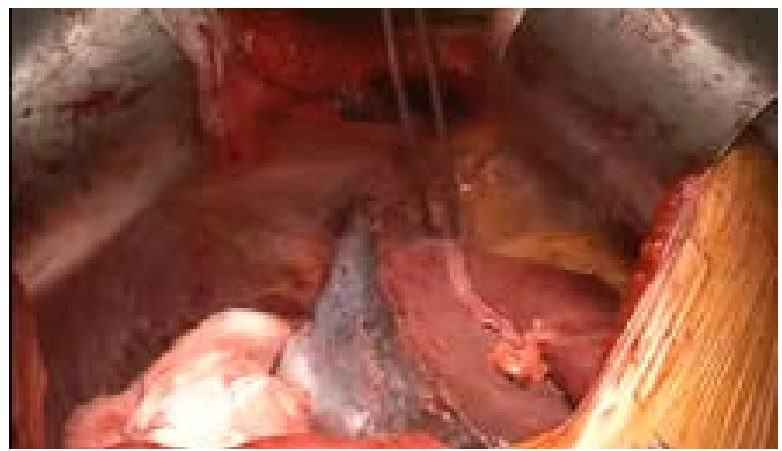

Fig 1: After completion of right hepatectomy. 


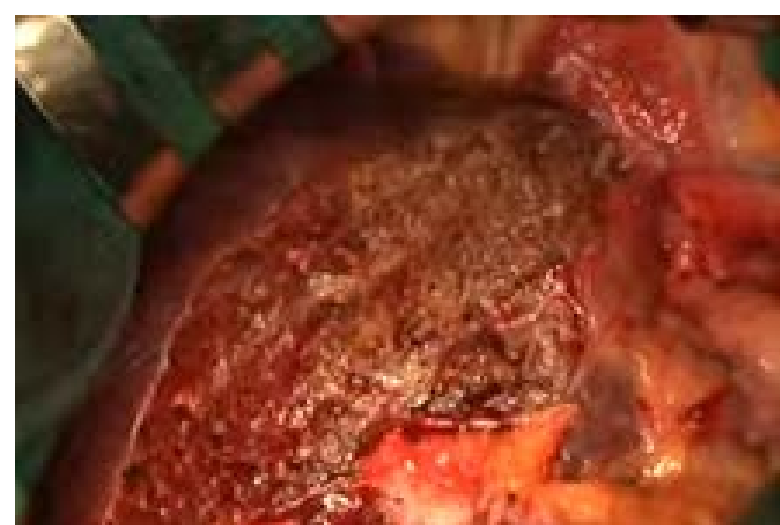

Fig 2: After completion of left hepatectomy.

The following parameters were compared between groups at baseline: Sex, age, chemotherapy, location and size of the primary tumor, number and maximal size of liver metastatic sites, and preoperative CEA levels. Median follow-up was 25 months (mean 29, range 6-83 months) for RFA and 34 months (mean 36, range 4-95 months) for the resection group. Kaplan-Meier curves were used to determine survival and the log-rank test was used for comparison between groups. Multivariate analysis was performed using the Cox Proportional Hazards Model. A P-value of $<0.05$ was accepted for statistical significance. The follow-up imaging studies included ultrasonography, CT, or MRI performed every 3 months after surgery for 1 year and every 6 months thereafter. The first follow-up imaging study was performed 1 month after RFA, and further follow-up studies were performed every 3 months for 1 year and every 6 months thereafter.

\section{Results}

This study included 40 patients, 28 males (70\%) and 12 females (30\%). Their ages ranged from 20 to 83 years. The mean metastatic site size was $2.3 \mathrm{~cm}$ (range, $1.9-3 \mathrm{~cm}$ ). Mean length of hospitalization was $1.2 \pm 0.4$ days for the RFA group and $6.1 \pm 0.4$ days for the HR group $(p<$ 0.001).

The demographic data of patients with colorectal liver metastasis and their tumor criteria were described in Table 1.

Twenty-four patients (60\%) died during the observation period, where 11 of them died from tumor progression, whereas 16 patients (40\%) remained alive at the end of that period.

In the RFA group, death was due to the progression of liver metastasis in 31\%, extra-hepatic disease in $26 \%$, and other unknown causes in $43 \%$. However, in the HR group, death was due to liver disease progression in $27 \%$, extra-hepatic disease progression in $41 \%$, and other unknown causes in $32 \%$. The main indication for referral to RFA included technical factors $(n=3)$, patient comorbidities $(n=9)$ and patients' decision $(n=4)$. The technical factors included various combinations of having fatty liver prejudicing adequate remnant liver function, obesity, the presence of nearby major vessel at risk of injury (inferior vena cava), and concomitant colorectal resection precluding a major liver resection at the same time. Although the groups were matched for age, gender, chemotherapy exposure, location and number or size of liver metastatic sites, the size of the primary tumor, or preoperative carcinoembryonic antigen level, RFA patients tended to have a higher ASA score (Table 1).

Table 1: Demographic data of patients with colorectal liver metastasis and their tumor criteria

\begin{tabular}{lccc}
\hline & HR $(\mathbf{n = 2 4 )}$ & RFA (n=16) & P value \\
\hline Number of males/females & $17 / 7$ & $11 / 5$ & 0.691 \\
Mean age (years) & 54.8 & 62.3 & 0.070 \\
Number of patients with chemotherapy (+/-) & $9 / 7$ & $9 / 7$ & 0.248 \\
Location of primary cancer (colon/rectum) & $7 / 17$ & $14 / 2$ & 0.437 \\
Mean size of primary cancer (cm) & 5.7 & 5.1 & 0.179 \\
Number of liver metastasis (single/multiple) & $15 / 9$ & $11 / 5$ & 0.599 \\
Mean level of preoperative CEA (ng/ml) & 45.89 & 24.78 & 0.749 \\
ASA score & {$[1-2] 16$ patients (67\%) } & {$[1-2] 7$ patients (44\%) } & 0.002 \\
& {$[3-4] 8$ patients (33\%) } & {$[3-4]$ patients (56\%) } &
\end{tabular}

The overall Kaplan-Meier median survival from the date of surgery was 53 months for HR patients and 29 months for RFA patients. The 3-year overall survival rates for patients in the HR and
RFA groups were 51.5 and $44.1 \%$, respectively $(p=0.285)$ (Figure 3). The median times of disease-free status for patients in the HR and RFA groups were 19 and 10 months, whereas the 
3-year disease-free survival rates were 33.1 and $27.2 \%$, respectively $(p=0.211)$ (Figure 4$)$. Thus, there were no significant differences between groups with respect to the overall or disease-free survival rates.

In the HR and RFA groups, 10 (41.6\%) and 7 $(43.7 \%)$ patients developed recurrence, respectively $(p=0.214)$. In the RFA group, repeated RFA was performed in three patients and HR was done in three patients for local recurrence in the follow-up. In one patient with local recurrence after RFA, neither RFA nor resection was performed due to multifocal liver recurrence. Sex, age, chemotherapy, location and size of the primary tumor, detection time of metastasis, number and size of liver metastatic sites, and preoperative CEA level was not significantly associated with the 3-year overall and disease-free survival rates. The complication rate was $1.25 \%(n=1)$ for RFA and $29.1 \%(n=7)$ for HR. In the RFA group, one patient $(5.9 \%)$ required prolonged inpatient care because of post operative fever of unknown reason, however, it might be from tumor necrosis and potential infection and nausea. The complications in the HR group included pulmonary complications $(n=7)$, wound infection $(n=4)$, bile leak $(2)$, ileus $(n=5)$, urinary retention $(n=3)$, and post-operative hemorrhage $(n=1)$.

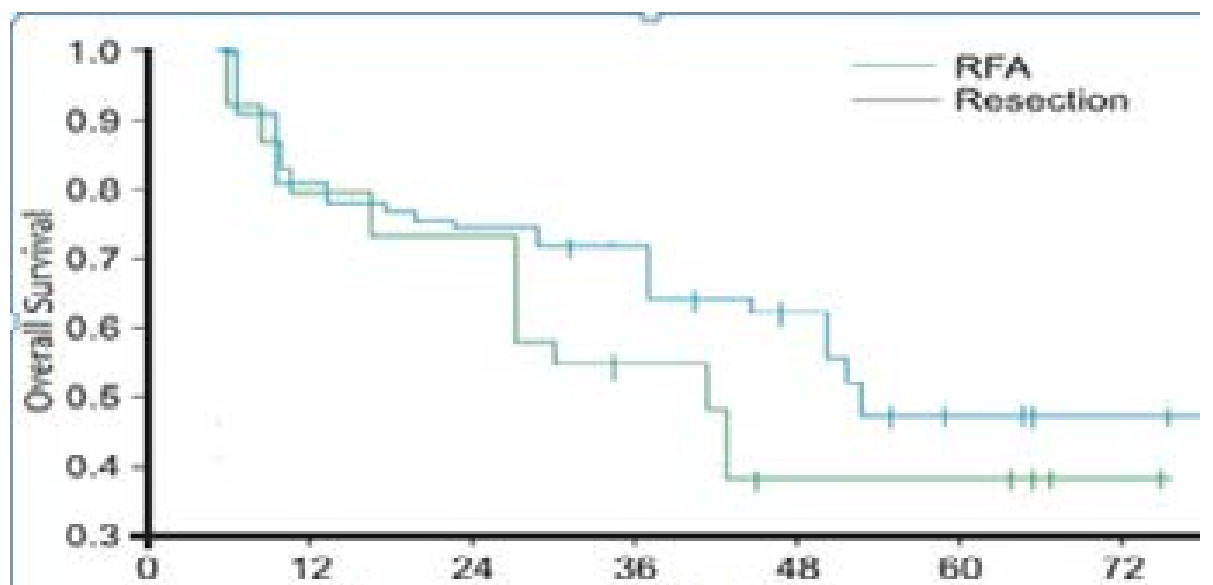

Fig 3: Overall Survival of patients with colorectal liver metastasis less than $3 \mathrm{~cm}$ treated by RFA and HR ( $p=0.285)$.

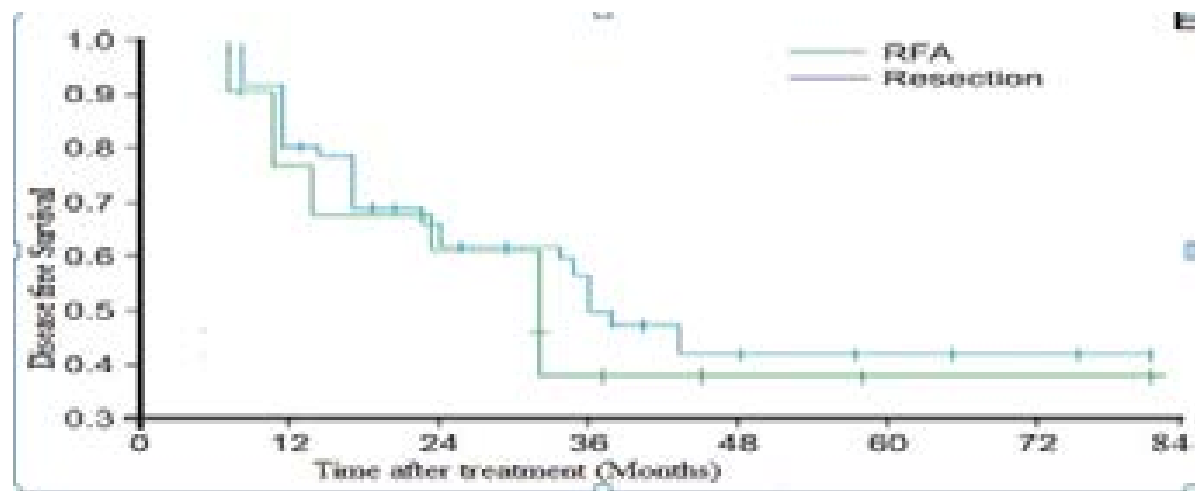

Fig 4: Disease-free survival of patients with colorectal liver metastasis less than $3 \mathrm{~cm}$ treated by RFA and HR ( $p=0.211)$.

\section{Discussion}

Although HR has been considered the standard modality for treatment of colorectal liver metastasis, ${ }^{10,11}$ only $10-25 \%$ of those patients were candidates for HR; whereas the others were not because of having systemic conditions, multiple liver metastases, and other problems. ${ }^{12,13}$
Thus, finding alternative treatments for those patients became necessary. This was first managed using cryotherapy for the liver metastasis, but RFA had replaced it due to its better patient's tolerance and tumor control with lower morbidity. ${ }^{14,15}$ RFA worked by using a high-frequency alternating current delivered through an electrode placed in 
the center of the tumor. ${ }^{8}$ That led to coagulative necrosis by raising the local tissue temperatures, to a level exceeding $100^{\circ} \mathrm{C}$, inducing parenchymal and tumor cell death.

The success of RFA had opened a question of whether it can be used for patients with resectable liver metastases. ${ }^{16,17}$ However, there were only a few studies to answer this question. It had been reported in one study that the overall median survival was 41 versus 37 months for $H R$ and RFA groups respectively, and their 3-year survival rates were $55.4 \%$ versus $52.6 \%$. On the other hand, other studies reported better 5 -year survival ( $71 \%$ versus $27 \%$ ) for HR versus RFA and overall median survival (56 versus 36 months) ${ }^{18}$ as well as disease-free survival ( 15 versus 8 months). Our study compared the therapeutic outcomes of $\mathrm{HR}$ and percutaneous RFA for resectable lesions of colorectal liver metastases, although the HR group had a slightly higher survival rate than the RFA group, the differences were not significant. The results indicate that RFA has comparable outcomes to those of HR with an overall survival of $51.5 \%$ for $H R$ versus $44.1 \%$ for RFA with $p=0.285$; and having a 3-year disease-free survival of 33.1 versus $25.2 \%$ respectively, with $p=0.211$.

In our study, co-morbidities were more likely to be found in RFA patients, however, our study compared HR to percutaneous RFA, which was known to have higher local recurrence rates compared to open or laparoscopic RFA. We reported in our study a complication rate of $1.25 \%(n=1)$ for RFA and $29.1 \%(n=7)$ for $H R$, these results were similar to those of other studies. As a result of recent technology, the outcomes of RFA have become comparable to those of HR. Oshowo et al. reported that the 3-year survival rate was almost the same for patients with solitary colorectal liver metastases treated with RFA (52.6\%) and for those who underwent HR (55.4\%). ${ }^{7}$ Meanwhile, in a study conducted on 102 with colorectal liver metastases that were offered RFA, Sørensen and co-workers reported that RFA had a 5-year survival rate of $44 \%$ which was comparable to that following HR. ${ }^{19}$ Similar studies reported that RFA was a safe alternative procedure for managing solitary colorectal liver metastases less than 3 $\mathrm{cm}$, with minimal morbidity and almost the same outcomes of HR. ${ }^{20,21}$ On the other hand, several studies reported that HR has better outcomes than RFA. Hur et al. analyzed 42 and 25 patients who underwent HR and RFA, respectively, for the treatment of solitary colorectal liver metastases; the 5 -year overall survival rate $(50.1 \%)$ and local recurrence-free survival rate $(89.7 \%)$ after HR were significantly higher than those after RFA (25.5 and $69.7 \%$, respectively). ${ }^{11}$ These results were consistent with outcomes reported by Lee and his colleagues in 2012.22 Meanwhile, in a study done by McKay et al. they reported that the 5-year survival rate was $43 \%$ after $\mathrm{HR}$ in comparison to $23 \%$ after RFA; whereas the 5 -year survival for patients with solitary lesions was $48 \%$ after HR and $15 \%$ after RFA. ${ }^{10}$ Other meta-analyses studies $^{23,24}$ further reported that HR is significantly superior to RFA for treatment of colorectal liver metastases. Weng and co-workers reported that postoperative morbidity is significantly higher $(24.1 \%)$ in patients that were operated upon by HR than those that were offered RFA (9.98). ${ }^{23}$ They stated that RFA should be offered for patients who were not suitable for HR and not to use it as a first-line option for treatment. However, it is worth mentioning that in these two meta-analyses, the RFA groups included patients with irresectable liver metastases; thus they experienced more severe disease progression.

In order to accurately report the results of the treatment outcomes for HR and RFA, another meta-analysis targeting studies involving patients receiving RFA as the only possible treatment are required. The major limitations of the present study were the lack of randomization of treatment modalities, the small numbers of patients, and the short follow-up duration. Because of small sample size, it seems that difference in survivals of both groups did not show statistical significance. Although RFA had been investigated as an alternative to surgery because of its safety and feasibility, the comparative effectiveness of RFA and HR has yet to be clearly demonstrated.

\section{Conclusion}

The 3-year overall and disease-free survival of patients with colorectal liver metastasis treated with RFA were comparable to those treated with HR. RFA could be considered as a primary treatment modality in particular cases when patients refuse surgery, with multiple co-morbidities or in cases of inadequate liver reserve. Therefore, larger multicenter studies with longer follow-up periods are required to yield more objectively applicable data to confirm the present results.

\section{References}

1. Wei AC, Greig PD, Grant D, Taylor B, Langer B, Gallinger S: Survival after hepatic resection for colorectal metastases: A 10 years' experience. Ann Surg Oncol 2006; 13: 668-676.

2. Abdalla EK, Vauthey JN, Ellis LM, Ellis V, Pollock $R$, Broglio KR, et al: Recurrence and outcomes following hepatic resection, radiofrequency ablation, and combined resection/ablation for colorectal liver metastases. Ann Surg 2004; 239: 818-827. 
3. Geoghegan JG, Scheele J: Treatment of colorectal liver metastases. Br J Surg 1999; 86: 158-169.

4. Fong $Y$, Fortner J, Sun RL, Brennan MF, Blumgart $\mathrm{LH}$ : Clinical score for predicting recurrence after hepatic resection for metastatic colorectal cancer: Analysis of 1001 consecutive cases. Ann Surg 1999; 230: 309-318.

5. Berber E, Rogers S, Siperstein A: Predictors of survival after laparoscopic radiofrequency thermal ablation of hepatocellular cancer: A prospective study. Surg Endosc 2005; 19: 710714.

6. Amersi FF, McElrath-Garza A, Ahmad A, Zogakis T, Allegra DP, Krasne R, et al: Longterm survival after radiofrequency ablation of complex unresectable liver tumors. Arch Surg 2006; 141: 318-326.

7. Oshowo A, Gillams A, Harrison E, Lees WR, Taylor I: Comparison of resection and radiofrequency ablation for treatment of solitary colorectal liver metastases. Br J Surg 2003; 90: 1240-1243.

8. Curley SA, Izzo F, Delrio P, Ellis LM, Granchi J, Vallone $P$, et al: Radiofrequency ablation of unresectable primary and metastatic hepatic malignancies: Results in 123 patients. Ann Surg 1999; 230: 1-8.

9. Gillams AR, Lees WR: Radiofrequency ablation of colorectal liver metastases. Abdom Imaging 2005; 30: 419-426.

10. McKay A, Fradette K, Lipschitz J: Long-term outcomes following hepatic resection and radiofrequency ablation of colorectal liver metastases. HPB Surg ID 2009; 346863: 1-8.

11. Hur H, Ko YT, Min BS, Kim KS, Choi JS, Sohn SK, et al: Comparative study of resection and radiofrequency ablation in the treatment of solitary colorectal liver metastases. Am J Surg 2008; 197: 728-736.

12. Fong Y, Fortner J, Sun RL, Brennan MF, Blumgart LH: Clinical score for predicting recurrence after hepatic resection for metastatic colorectal cancer: Analysis of 1001 consecutive cases. Ann Surg 1999; 230: 309-318.

13. Silen W: Hepatic resection for metastases from colorectal carcinoma is of dubious value. Arch Surg 1989; 124: 1021-1022.

14. Curley SA, Izzo F, Ellis LM, Nicolas Vauthey J, Vallone P: Radiofrequency ablation of hepatocellular cancer in 110 patients with cirrhosis. Ann Surg 2000; 232: 381-391.

15. Siperstein A, Garland A, Engle K, Rogers S, Berber E, Foroutani A, et al: Local recurrence after laparoscopic radiofrequency thermal ablation of hepatic tumors. Ann Surg Oncol 2000;7:106-113.

16. Siperstein AE, Berber $E$, Ballem N, Parikh RT: Survival after radiofrequency ablation of colorectal liver metastases: 10-year experience. Ann Surg 2007; 246: 559-565.

17. Machi J, Oishi AJ, Sumida K, Sakamoto K, Furumoto $\mathrm{NL}$, Oishi $\mathrm{RH}$, et al: Long-term outcome of radiofrequency ablation for unresectable liver metastases from colorectal cancer: Evaluation of prognostic factors and effectiveness in first- and second-line management. Cancer J 2006; 12: 318-326.

18. Park IJ, Kim HC, Yu CS, Kim PN, Won HJ, Kim JC: Radiofrequency ablation for metachronous liver metastasis from colorectal cancer after curative surgery. Ann Surg Oncol 2007; 15: 227-232.

19. Sørensen SM, Mortensen FV, Nielsen DT: Radiofrequency ablation of colorectal liver metastases: Long-term survival. Acta Radiol 2007; 48: 253-258.

20. Kim KH, Yoon YS, Yu CS, Kim TW, Kim HJ, Kim PN, et al: Comparative analysis of radiofrequency ablation and surgical resection for colorectal liver metastases. J Korean Surg Soc 2011; 81: 25-34.

21. Gillams AR, Lees WR: Five-year survival following radiofrequency ablation of small, solitary, hepatic colorectal metastases. J Vasc Interv Radiol 2008; 19: 712-717.

22. Lee $\mathrm{KH}$, Kim HO, YooCH, Son BH, Park $\mathrm{YL}$, Cho YK, et al: Comparison of radiofrequency ablation and resection for hepatic metastasis from colorectal cancer. Korean J Gastroenterol 2012; 59: 218-223.

23. Weng $M$, Zhang $Y$, Zhou $D$, Yang $Y$, Tang $Z$, Zhao M, et al: Radiofrequency ablation versus resection for colorectal cancer liver metastases: A meta-analysis. Open Access 2012; 7: 1-8.

24. .Wu YZ, Li B, Wang T, Wang SJ, Zhou YM: Radiofrequency ablation versus hepatic resection for solitary colorectal liver metastasis: a meta-analysis. World J 2011; 17: 4143-4148. 